\title{
Fully decomposable split graphs
}

\author{
Hajo Broersma ${ }^{\mathrm{a}, 1}$, Dieter Kratsch ${ }^{\mathrm{b}}$, Gerhard J. Woeginger ${ }^{\mathrm{c}}$ \\ a Faculty of Electrical Engineering, Mathematics and Computer Science, University of Twente, P.O. Box 217, \\ 7500 AE Enschede, The Netherlands \\ ${ }^{\mathrm{b}}$ LITA, Université Paul Verlaine - Metz, 57045 Metz Cedex 01, France \\ ${ }^{\mathrm{c}}$ Department of Mathematics and Computer Science, TU Eindhoven, P.O. Box 513, 5600 MB Eindhoven, The Netherlands
}

\section{A R T I C L E I N F O}

\section{Article history:}

Available online 24 August 2012

\begin{abstract}
A B S T R A C T
We discuss various questions around partitioning a split graph into connected parts. Our main result is a polynomial time algorithm that decides whether a given split graph is fully decomposable, that is, whether it can be partitioned into connected parts of orders $\alpha_{1}, \alpha_{2}, \ldots, \alpha_{k}$ for every $\alpha_{1}, \alpha_{2}, \ldots, \alpha_{k}$ summing up to the order of the graph. In contrast, we show that the decision problem whether a given split graph can be partitioned into connected parts of orders $\alpha_{1}, \alpha_{2}, \ldots, \alpha_{k}$ for a given partition $\alpha_{1}, \alpha_{2}, \ldots, \alpha_{k}$ of the order of the graph, is NP-hard.
\end{abstract}

(c) 2012 Elsevier Ltd. All rights reserved.

\section{Introduction}

Throughout we only consider finite undirected graphs without loops or multiple edges. Let $G=(V, E)$ be a graph on $n$ vertices, and let $\alpha=\left(\alpha_{1}, \ldots, \alpha_{k}\right)$ denote a partition of $n$, that is, a sequence of positive integers $\alpha_{1}, \ldots, \alpha_{k}$ with $\sum_{i=1}^{k} \alpha_{i}=n$. The graph $G$ is called $\alpha$-decomposable if there exists a partition of $V$ into disjoint subsets $A_{1}, \ldots, A_{k}$ of cardinality $\left|A_{i}\right|=\alpha_{i}$ for $1 \leq i \leq k$ such that every set $A_{i}$ induces a connected subgraph of $G$. Such a partition is called an $\alpha$-decomposition of $G$, and a (connected) subgraph induced by $\left|A_{i}\right|=\alpha_{i}$ vertices is also referred to as an $\alpha_{i}$-component of the $\alpha$-decomposition. A graph is called fully decomposable (or arbitrarily vertex decomposable) if it is $\alpha$-decomposable for every partition $\alpha$ of $n$.

Fully decomposable graphs were introduced by Horňák and Woźniak [7]. The concept of full decomposability can be considered as lying somewhere between hamiltonicity and factorability: if

\footnotetext{
An extended abstract was presented at IWOCA 2009 and has appeared in LNCS [3].

E-mail addresses: h.j.broersma@utwente.nl, hajo.broersma@durham.ac.uk (H. Broersma), kratsch@univ-metz.fr (D. Kratsch), gwoegi@win.tue.nl (G.J. Woeginger).

1 Tel.: +31534893443. 
a graph $G$ has a Hamiltonian path, that is, a path containing all vertices of $G$, then $G$ is obviously fully decomposable; on the other hand a fully decomposable graph on an even number of vertices contains a 1-factor, that is, a spanning 1-regular subgraph. Deciding whether a graph has a Hamiltonian path is a well-known NP-hard problem, whereas deciding whether a graph has a 1-factor is known to be solvable in polynomial time.

Here we consider the following two natural algorithmic questions centered around $\alpha$ decompositions of graphs. We formulate these questions as decision problems, as follows.

Q1: Decide whether a given graph $G$ is $\alpha$-decomposable for a given partition $\alpha$.

Q2: Decide whether a given graph $G$ is fully decomposable.

Question Q1 is notoriously hard. Dyer and Frieze [4] proved that it is NP-hard to decide whether a planar graph is $(3,3, \ldots, 3)$-decomposable. Barth and Fournier [2] showed that Q1 is NP-hard for trees. Generally speaking, Q1 seems to be NP-hard for every natural non-trivial class of specially structured graphs.

The computational complexity of Question Q2 is not understood. We are aware of only a single result on Question Q2 from the literature: Barth et al. [1] designed a polynomial time algorithm for deciding whether a given tripode (a tree with a single vertex of degree three, and all other vertices of degree one or two) is fully decomposable. Barth and Fournier [2] also proved that every fully decomposable tree has maximum vertex degree at most four. Determining the precise computational complexity of Q2 is an outstanding open problem: the problem is neither known to be NP-hard, nor is it known to be contained in the class NP.

\section{Results of this paper}

In this paper we restrict our questions to split graphs. A graph $G=(V, E)$ with vertex set $V$ and edge set $E$ is a split graph (see for instance [6]) if its vertex set can be partitioned into an induced independent set $I$, that is, a set of mutually nonadjacent vertices, and a clique $C$, that is, a set of mutually adjacent vertices. Often split graphs are specified in the form $G=(C, I, E)$.

In this paper, we will resolve the computational complexity of Questions Q1 and Q2 for split graphs. The following two theorems show that for this graph class Q1 is hard, whereas Q2 is easy.

Theorem 1. It is NP-hard to decide whether a given split graph with $n$ vertices is $\alpha$-decomposable for a given partition $\alpha$ of $n$.

Theorem 2. It can be decided in polynomial time whether a given split graph with $n$ vertices is fully decomposable.

Theorem 1 will be proved in the following Section 3, and Theorem 2 will be proved in the remaining part of this paper.

\section{The hardness proof}

In this section we will prove Theorem 1 . The reduction is done from the following version of the NP-hard $d$-DIMENSIONAL MATCHING problem (see [5]).

PROBLEM: $d$-DIMENSIONAL MATCHING $(d$-DM)

INPUT: A ground set $X=\left\{x_{1}, \ldots, x_{q d}\right\}$ of $q d$ elements; a family $\&$ of $d$-element subsets $S_{1}, \ldots, S_{\ell}$ of $X$.

QUESTION: Can set $X$ be partitioned into $q$ disjoint subsets from $S_{1}, \ldots, S_{\ell}$ ?

For an instance of this problem $d$-DM, we now construct the following corresponding split graph which is sketched in Fig. 1.

- For every element $x \in X$, the independent set $I$ contains a corresponding vertex $i(x)$. Furthermore, in the independent set $I$ there are $\ell-q$ groups $D_{1}, \ldots, D_{\ell-q}$ of dummy vertices; every such group consists of exactly $d-1$ vertices. 


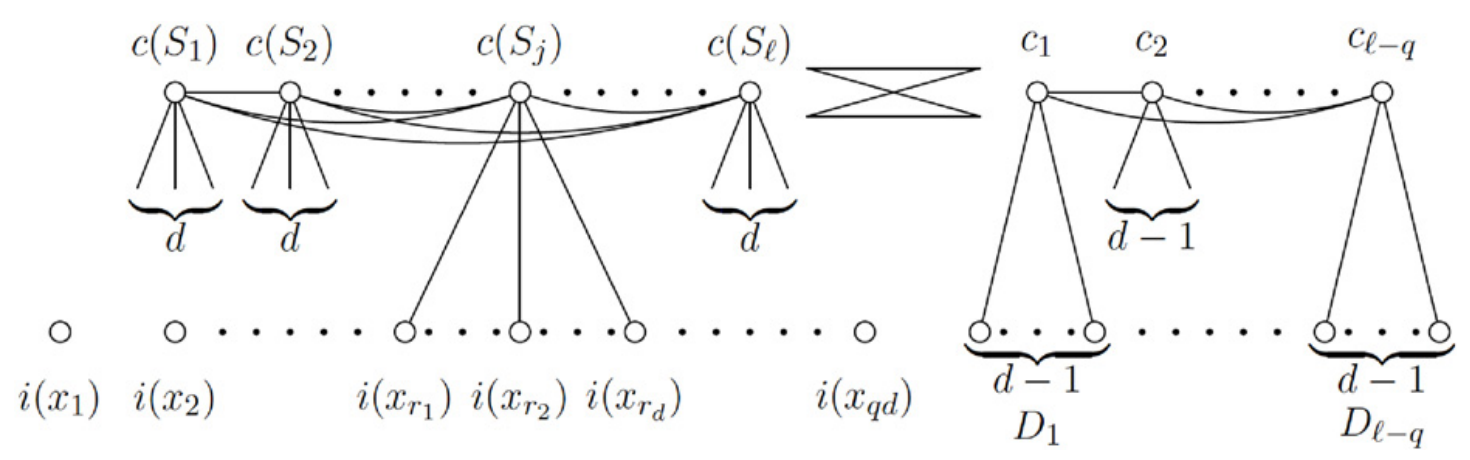

Fig. 1. The split graph in the proof of Theorem 1 .

- For every set $S$ in the family $\&$, the clique $C$ contains a corresponding vertex $c(S)$. Furthermore, the clique $C$ contains $\ell-q$ dummy vertices $c_{1}, \ldots, c_{\ell-q}$.

- There is an edge between any two vertices in the clique $C$.

- Whenever $x \in S$ for some $x \in X$ and some $S \in \delta$, there is an edge between $i(x)$ and $c(S)$.

- Furthermore, for $k=1, \ldots, \ell-q$ the dummy vertex $c_{k}$ is joined to the $d-1$ dummy vertices in the group $D_{k}$.

The resulting split graph $G$ has $2 \ell-q$ vertices in $C$, has $(d-1) \ell+q$ vertices in $I$, and thus consists altogether of $(d+1) \ell$ vertices. Finally, we define the vector $\alpha=(d+1, d+1, \ldots, d+1)$ that consists of $\ell$ components of value $d+1$. We claim that the split graph $G$ is $\alpha$-decomposable if and only if the instance of $d$-DM has answer YES.

First assume that the instance of $d$-DM has answer YES. Consider the partition of $X$ into $q$ subsets from $\$$. For every set $S$ occurring in this partition, we put vertex $c(S)$ together with all vertices $i(x)$ with $x \in S$ into one connected component. For every set $S$ not occurring in this partition, we put vertex $c(S)$ together with one of the dummy vertices $c_{k}$ and the vertices in the group $D_{k}$ into one connected component. This yields that $G$ is $\alpha$-decomposable.

Next assume that the graph $G$ is $\alpha$-decomposable. Every connected component in the decomposition has order $d+1$ and hence contains at least one vertex of $C$. Every dummy vertex $c_{k}$ must be in the same connected component with the vertices in group $D_{k}$, and with exactly one of the vertices $c(S)$. Since there are only $q$ of the vertices $c(S)$ left, each of the $q$ remaining connected components contains exactly one of the vertices $c(S)$. This corresponds to the desired partition of the set $X$.

This completes the NP-hardness argument, and the proof of Theorem 1. Since $d$-DM is NP-hard for every fixed $d \geq 3$, we have actually established the following stronger statement.

Corollary 3. For every fixed integer $f \geq 4$, it is NP-hard to decide whether a given split graph on qf vertices is $\alpha$-decomposable with respect to the vector $\alpha=(f, f, \ldots, f)$ consisting of $q$ components of value $f$.

The following sections will show that the statement in Corollary 3 is essentially strongest possible. For $f \leq 3$, the corresponding decomposition problem allows a polynomial time solution.

In the above NP-hardness proof, in the graph that is sketched in Fig. 1, the dummy vertices in the groups $D_{1}, \ldots, D_{\ell-q}$ are all of degree 1 . Does the problem perhaps become easier, if we additionally impose a degree condition and forbid vertices of low degree? We will now show that the problem remains NP-hard also under such degree conditions.

The argument is a simple modification of the above construction, and only adds some small piece to it. Let $\delta \geq 1$ denote the desired lower bound on the minimum degree. We create $\delta$ new clique vertices together with $d \cdot \delta$ new vertices in the independent set, and we add the complete bipartite graph between these two new vertex sets to the construction. Furthermore, every old vertex in the independent set is joined to every new clique vertex. Every vertex in the resulting graph has degree at least $\delta$. It can be seen that every feasible partition into connected components of order $d+1$ will group the new vertices together: the only feasible way of connecting the $d \cdot \delta$ new vertices in the independent 
set is by dividing them into $\delta$ groups of cardinality $d$ and by adding one new clique vertex to each such group. This completes the proof of the following statement.

Corollary 4. Let $\delta \geq 1$ be an integer. It is NP-hard to decide whether a given split graph on $n$ vertices with minimum degree $\delta$ is $\alpha$-decomposable for a given partition $\alpha$ of $n$.

\section{Primitive partitions}

The special structure of split graphs enables us to focus on very specific partitions into small parts in order to prove Theorem 2.

For $n \geq 2$, a partition $\alpha$ of $n$ is called 2-3-primitive if it is of one of the following forms.

- $\alpha=(1,3,3, \ldots, 3)$ consists of threes and a single one;

- $\alpha=(2, \ldots, 2,3,3, \ldots, 3)$ only consists of twos and threes.

The following lemma shows that for analyzing the full decomposability of a split graph, we can restrict our attention to $2-3$-primitive partitions.

Lemma 5. A split graph on $n$ vertices is fully decomposable if and only if it is $\alpha$-decomposable for every 2-3-primitive partition $\alpha$ of $n$.

Proof. The only-if-statement is implicit in the definition of a fully decomposable graph. For the ifstatement, we recall that every integer $\ell \geq 2$ can be written in the form $\ell=2 a+3 b$ with nonnegative integers $a$ and $b$. Consider an arbitrary partition $\alpha=\left(\alpha_{1}, \ldots, \alpha_{k}\right)$ of $n$. Replace every $\alpha_{i} \geq 2$ in $\alpha$ by a partition of $\alpha_{i}$ into $a_{i}$ twos and $b_{i}$ threes. Let $\alpha_{0}$ denote the number of ones in the vector $\alpha$. If $\alpha_{0} \geq 2$, then replace the ones in vector $\alpha$ by a partition of $\alpha_{0}$ into $a_{0}$ twos and $b_{0}$ threes. If $\alpha_{0}=1$ and there is a two in the partition, then replace the one and a two by a three; otherwise leave the one as it is. The resulting new partition $\alpha^{\prime}=\left(\alpha_{1}^{\prime}, \ldots, \alpha_{m}^{\prime}\right)$ of $n$ is of the form $(1,3,3, \ldots, 3)$ or $(2, \ldots, 2,3,3, \ldots, 3)$, and hence 2-3-primitive. By assumption the split graph $G$ is $\alpha^{\prime}$-decomposable. We let $A_{1}^{\prime}, \ldots, A_{m}^{\prime}$ denote the corresponding connected vertex sets. Every set $A_{j}^{\prime}$ with $\alpha_{j}^{\prime}=\left|A_{j}^{\prime}\right| \geq 2$ contains at least one clique-vertex; therefore, the union of the $a_{i}$ two-element sets and the $b_{i}$ threeelement sets corresponding to component $\alpha_{i}$ is a connected vertex set $A_{i}$ with $\alpha_{i}$ elements. In case $\alpha_{0}=1$ and the one was combined with a two, it is obvious how to split off a vertex in order to leave a 2-component. This yields that $G$ is $\alpha$-decomposable.

We note that Lemma 5 already implies an NP-certificate for deciding whether an $n$-vertex split graph is fully decomposable: the certificate lists all 2-3-primitive partitions of $n$ together with the corresponding decompositions into connected parts. The following sections contain even stronger results and yield a proof of Theorem 2 .

\section{Canonical primitive partitions}

Next let us introduce canonical primitive partitions as a crucial subfamily of the 2-3-primitive partitions. Let $n \geq 2$ be an integer.

- If $n=2 k$ is even, then the canonical 2-primitive partition of $n$ consists of $k$ twos.

If $n=2 k+1$ is odd, then the canonical 2-primitive partition of $n$ consists of $k-1$ twos and a single three.

- If $n=3 k$, then the canonical 3-primitive partition of $n$ consists of $k$ threes.

If $n=3 k+1$, then the canonical 3-primitive partition of $n$ consists of $k$ threes and a single one. If $n=3 k+2$, then the canonical 3-primitive partition of $n$ consists of $k$ threes and a single two.

The following lemma strengthens the statement of Lemma 5.

Lemma 6. A split graph with $n$ vertices is fully decomposable if and only if it is $\alpha$-decomposable for the canonical 2-primitive partition $\alpha$ of $n$ and for the canonical 3-primitive partition $\alpha$ of $n$. 

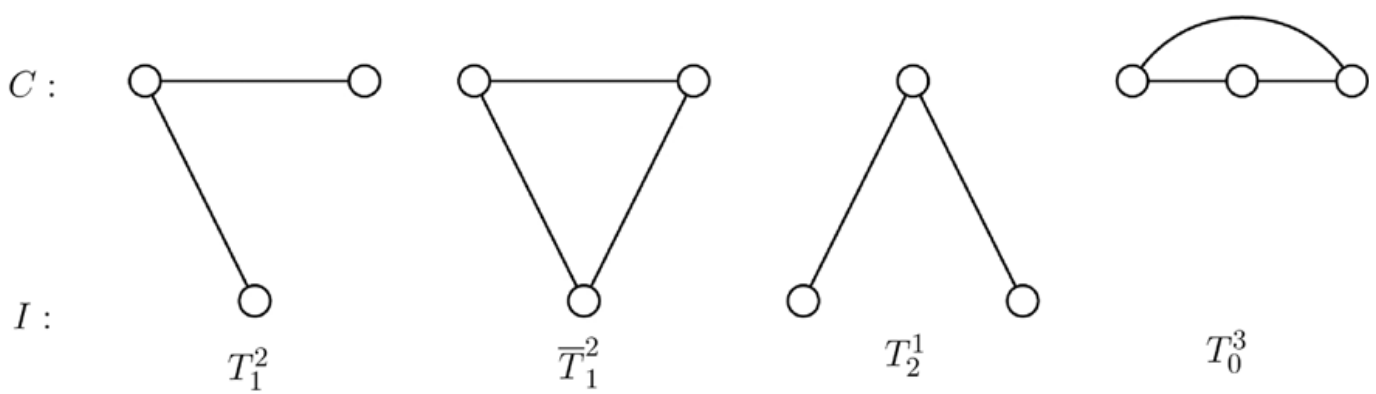

Fig. 2. The possible 3-components in a split graph.

The rest of this section is dedicated to the proof of Lemma 6. We first introduce some additional notation and terminology.

We use $2^{r} 3^{s}$ to denote a partition of $n=2 r+3 s$ into $r$ (possibly $r=0$ ) twos and $s$ (possibly $s=0$ ) threes. A partition of $n=3 k+1$ into $k$ threes and 1 one is denoted by $13^{k}$.

Suppose $G=(C, I, E)$ is a split graph and $H$ is a subgraph of $G$. Then a vertex of $V(H) \cap C$ or $V(H) \cap I$ is called a $C$-vertex or $I$-vertex of $H$, respectively. Analogously, we call a neighbor $u$ of a vertex $v \in V(G)$ a $C$-neighbor or I-neighbor of $v$ if $u \in C$ or $u \in I$, respectively. If $|V(H)|=3$, we say that $H$ is a $T_{i}^{c}$ if $|V(H) \cap C|=c$ and $|V(H) \cap I|=i$; in the special case that $c=2$ and $i=1$ we add a bar (only) if $T_{1}^{2}$ is a triangle, so we use $\bar{T}_{1}^{2}$ instead of $T_{1}^{2}$ if and only if the three vertices induce a triangle in $G$. In Fig. 2 we illustrate the possible 3-components in a split graph.

For proving Lemma 6 it is sufficient to prove the following result.

Lemma 7. If a split graph $G$ with $n$ vertices is $\alpha$-decomposable for the canonical 2-primitive partition $\alpha$ of $n$ and for the canonical 3-primitive partition $\alpha$ of $n$, then $G$ is $\alpha$-decomposable for every 2-3-primitive partition $\alpha$ of $n$.

Proof. Let $G=(C, I, E)$ be a split graph on $n$ vertices, and assume that $G$ is $\alpha$-decomposable for the canonical 2-primitive partition $\alpha$ of $n$ and for the canonical 3-primitive partition $\alpha$ of $n$. First note that we may assume that $n \geq 10$; if $n<10$, then the only possible 2-3-primitive partitions are the canonical 2-primitive and the canonical 3-primitive partitions. Second, note that $G$ has a matching saturating at least $|I|-1$ vertices of $I$ (and all vertices of $I$ if $n$ is even); since $I$ is an independent set, this follows immediately from the hypothesis that $G$ is $\alpha$-decomposable for the canonical 2-primitive partition $\alpha$ of $n$. This also implies that $|C| \geq|I|-1$.

Definition 8. We say that $G$ is $(3,3)$-reducible if and only if it has the following property: If $G$ is $2^{r} 3^{s}$-decomposable for some $r \geq 0$ and $s \geq 4$, then it is also $2^{r+3} 3^{s-2}$-decomposable.

Similarly, we say that $G$ is $(1,3)$-reducible if and only if $G$ has the following property: If $G$ is $13^{k}$-decomposable for some $k \geq 3$, then it is also $2^{2} 3^{k-1}$-decomposable.

We claim that in the language of this definition, it is now sufficient to prove that $G$ is both $(3,3)$ reducible and $(1,3)$-reducible. First of all, notice that the 2-3-primitive partition $13^{k}$ is already canonical 3-primitive, and that the 2-3-primitive partition $2^{r} 3^{s}$ is canonical primitive whenever $r \leq 1$ or $s \leq 1$. Suppose $r \geq 2$ and $s \geq 2$. If $r=2$, we can obtain a decomposition from the decomposition for the canonical primitive partition $13^{s+1}$ if $G$ is $(1,3)$-reducible. If $r \geq 3$, we can obtain a decomposition from the decomposition for the 2-3-primitive partition $2^{r-3} 3^{s+2}$ if $G$ is $(3,3)$-reducible. The latter is canonical primitive if $r \leq 4$ and can be treated in a similar way if $r \geq 5$. Hence it is sufficient to prove that $G$ is both $(3,3)$-reducible and $(1,3)$-reducible.

The following two claims establish these facts, and thus complete the proof of Lemma 7.

Claim 9. $G$ is $(3,3)$-reducible.

Proof. Suppose $G$ has a $2^{r} 3^{s}$-decomposition $\alpha$ with $r \geq 0$ and $s \geq 4$. Then at least two of the 3-components in $\alpha$ have at least two $C$-vertices since $|C| \geq|I|-1$. It is obvious how to decompose the subgraph of $G$ induced by the six vertices of two such 3-components into three 2-components. 

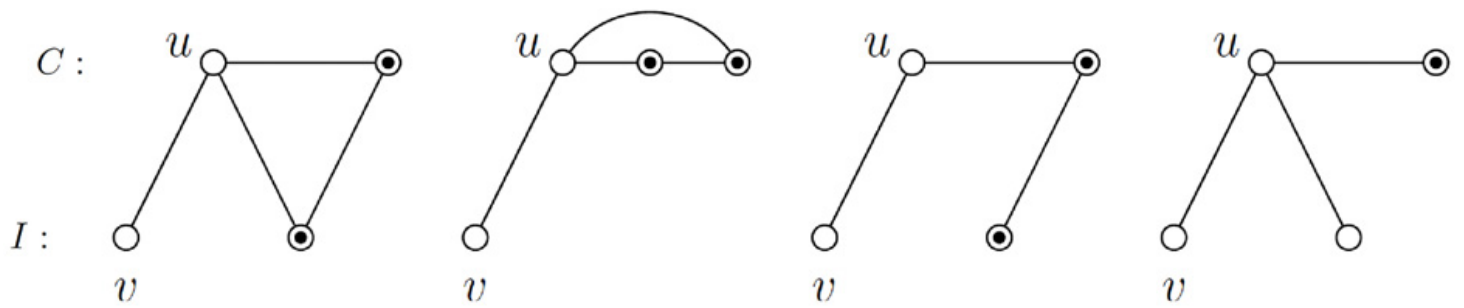

Fig. 3. The four cases with $v \in I$ and $u$ not the $C$-vertex of a $T_{2}^{1}$.

Claim 10. $G$ is $(1,3)$-reducible.

Proof. Suppose $G$ has a $13^{k}$-decomposition $\alpha$ with $k \geq 3$. Then at least one of the 3-components in $\alpha$ has at least two $C$-vertices since $|C| \geq|I|-1$. Let $H$ denote such a 3-component, and let $v$ denote the vertex of the 1-component in $\alpha$.

If $v \in C$ it is clear how to decompose the subgraph of $G$ induced by $V(H) \cup\{v\}$ into two 2-components.

Next suppose $v \in I$. Clearly, $v$ is not an isolated vertex since $G$ is $\alpha$-decomposable for the canonical 2-primitive partition $\alpha$ of $n$. Let $u$ be a $C$-neighbor of $v$. If $u$ is in a $\bar{T}_{1}^{2}$ or $T_{0}^{3}$ of $\alpha$, or if it is the vertex with degree 1 in a $T_{1}^{2}$ of $\alpha$, then it is again clear how to decompose the subgraph of $G$ induced by $v$ and the vertices of the 3-component containing $u$ into two 2-components.

If $u$ is the vertex with degree 2 in a $T_{1}^{2}$ of $\alpha$, we use that $\alpha$ contains at least one other 3-component $H^{\prime}$ with at least two $C$-vertices since $|C| \geq|I|-1$ and $v \in I$. In this case we can combine $v$ with $u$ and its $I$-neighbor in $T_{1}^{2}$ into a 3-component, and we can decompose the subgraph of $G$ induced by the remaining vertex of this $T_{1}^{2}$ and the vertices of $H^{\prime}$ into two 2-components.

A similar transformation along a longer chain of 3-components can be used in the remaining case where all the $C$-neighbors of $v$ are the $C$-vertex of a $T_{2}^{1}$. We fix a matching $M$ saturating at least $|I|-1$ vertices of $I$. Suppose that $v u \notin M$ for all possible choices of $u$. If $u$ is adjacent to some other vertex $v^{\prime}$ by an edge of $M$, we replace the edge $u v^{\prime}$ by the edge $u v$ in $M$; if $u$ is not incident with an edge of $M$, we add $u v$ to $M$. Hence we may assume that $u v \in M$ for a $C$-vertex $u$ of a $T_{2}^{1}$. At least one of the other vertices $v^{\prime}$ of this $T_{2}^{1}$ is incident with an edge of $M$ joining it to a $C$-vertex $u^{\prime} \neq u$. If $u^{\prime}$ is not the $C$-vertex of a $T_{2}^{1}$, we can take $v^{\prime}$ and $u^{\prime}$ instead of $v$ and $u$ and the $T_{2}^{1}$ consisting of $u, v$ and the third vertex $x \neq u, v^{\prime}$ of the original $T_{2}^{1}$, and treat this case as before. In the other case $u^{\prime}$ is the $C$-vertex of a $T_{2}^{1}$ and we can continue in the same way with an edge of $M$ joining one of the neighbors of $u^{\prime}$ in the $T_{2}^{1}$ to a new vertex $u^{\prime \prime} \neq u, u^{\prime}$, etc. By the fact that $G$ is a finite graph and since $|C| \geq|I|-1$, there is an alternating path $P=v_{1} v_{2} \ldots v_{2 t}$ starting at $v=v_{1}$ and terminating at a vertex $w=v_{2 t}$ in a $\bar{T}_{1}^{2}, T_{1}^{2}$ or $T_{0}^{3}$, in which each $v_{2 j}$ with $1 \leq j<t$ is the $C$-vertex of a $T_{2}^{1}$ and each $v_{2 j+1}$ with $1 \leq j<t$ is an $I$-vertex adjacent to $v_{2 j}$ in the corresponding $T_{2}^{1}$ for $t-1$ disjoint 3-components isomorphic to $T_{2}^{1}$. The chain of these $t-1$ copies of a $T_{2}^{1}$ without the vertex $v_{2 t-1}$ together with the vertex $v$ and the edges $v_{2 j-1} v_{2 j}$ with $1 \leq j<t$ can be transformed into $t-1$ new $T_{2}^{1}$ s by swapping the edges of $P$ (meaning that we include all edges $v_{2 j-1} v_{2 j}$ with $1 \leq j<t$ and remove all edges $v_{2 j} v_{2 j+1}$ with $1 \leq j<t$ ).

The remaining vertex $v_{2 t-1}$ and the 3 -component $H_{w}$ containing $w$ can be treated as before, yielding a decomposition of the subgraph of $G$ induced by $V\left(H_{w}\right) \cup\left\{v_{2 t-1}\right\}$ into two 2-components in case $w$ is not the vertex with degree 2 in a $T_{1}^{2}$; otherwise we use again that $\alpha$ contains at least one other 3-component $H^{\prime}$ with at least two $C$-vertices. In this case we can combine $v_{2 t-1}$ with $w$ and its $I$-neighbor in $T_{1}^{2}$ into a 3-component, and we can decompose the subgraph of $G$ induced by the remaining vertex of this $T_{1}^{2}$ and the vertices of $H^{\prime}$ into two 2-components (see Figs. 3 and 4).

This completes the proof of the lemma.

\section{The polynomial time result}

From the previous sections we conclude that to prove Theorem 2 it now suffices to prove the following two lemmas. The first lemma can be proved by straightforward matching techniques, but 


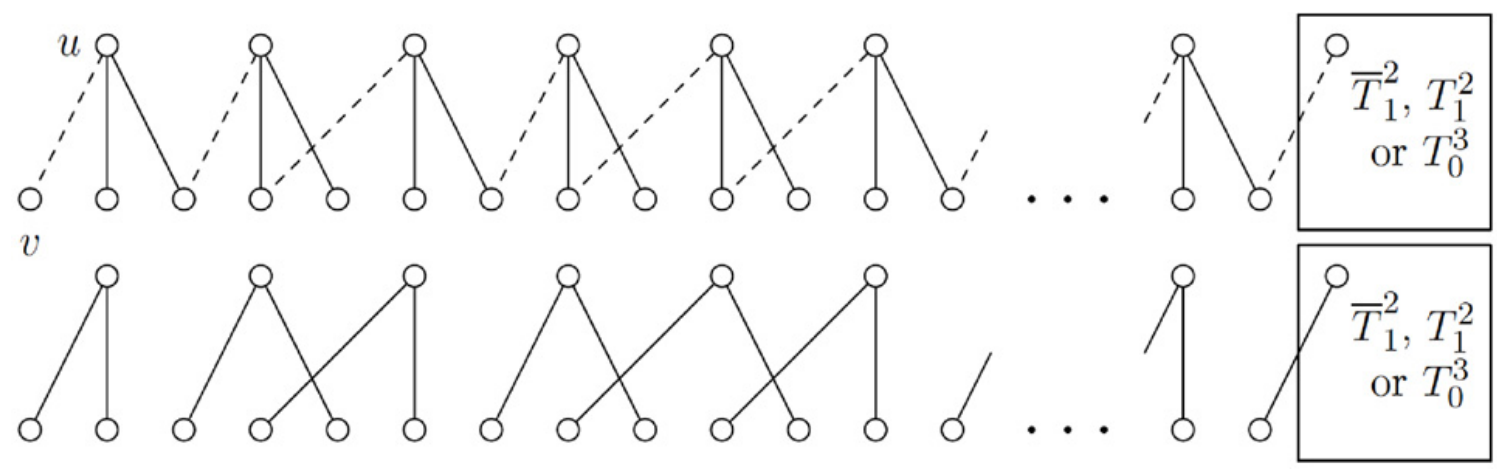

Fig. 4. The case with $v \in I$ and $u$ the $C$-vertex of a $T_{2}^{1}$.

for the second lemma we have to apply a more sophisticated technique closely related to matching theory.

Lemma 11. Let $G=(V, E)$ be a split graph on $n$ vertices, and let $\alpha$ be the canonical 2-primitive partition of $n$. Then it can be decided in polynomial time whether $G$ is $\alpha$-decomposable.

Proof. This boils down to a bipartite matching problem. If $n$ is even, we need to find a matching from the independent set $I$ into the clique $C$. If $n$ is odd, then we check all possibilities for the extra component with three vertices.

Lemma 12. Let $G=(V, E)$ be a split graph on $n$ vertices, and let $\alpha$ be the canonical 3-primitive partition of $n$. Then it can be decided in polynomial time whether $G$ is $\alpha$-decomposable.

Proof. Our main tool is the following result from matching theory (see for instance [8]). Let $G^{\prime}=\left(V^{\prime}, E^{\prime}\right)$ be an edge-weighted graph, and for every vertex $v \in V^{\prime}$ let $d(v)$ be a non-negative integer. Then we can determine in polynomial time a maximum-weight subset $F^{\prime} \subseteq E^{\prime}$ of the edges, such that in the graph $\left(V^{\prime}, F^{\prime}\right)$ every vertex $v$ has degree $d(v)$, or find out that no such set $F^{\prime}$ exists.

Consider a split graph $G=(C, I, E)$. We only discuss the case where the number of vertices is of the form $n=3 k$; the other cases can be handled by checking all possibilities for the extra component with one or two vertices. We construct an auxiliary graph $G^{\prime}=\left(V^{\prime}, E^{\prime}\right)$.

- The graph $G^{\prime}$ contains all vertices in $C \cup I$, together with all edges in $E$ between $C$ and $I$. All these edges have weight 0.

- For every vertex $v \in C$, the graph $G^{\prime}$ contains two additional vertices $v^{\prime}$ and $v^{\prime \prime}$. The three vertices $v, v^{\prime}, v^{\prime \prime}$ form a triangle. The weight of the edge $v^{\prime} v^{\prime \prime}$ is 1 , and the weight of the other two edges is 0 .

- There is a special vertex $v^{*}$ that is adjacent to all vertices in $C$. All edges between $v^{*}$ and $C$ have weight -1 .

For $0 \leq s \leq k$, we define an instance $G_{s}^{\prime}$ of the above matching problem, as sketched in Fig. 5 . The underlying edge-weighted graph is $G^{\prime}$, and the values $d(v)$ are defined as follows.

- For every vertex $v \in I$, we set $d(v)=1$. For every vertex $v \in C$, we set $d(v)=2$ and $d\left(v^{\prime}\right)=d\left(v^{\prime \prime}\right)=1$. Finally, we set $d\left(v^{*}\right)=s$.

We claim that the considered split graph $G=(C, I, E)$ is $\alpha$-decomposable for $\alpha=(3,3, \ldots, 3)$ if and only if at least one of these graphs $G_{s}^{\prime}$ (with $0 \leq s \leq k$ ) possesses a subgraph $\left(V^{\prime}, F^{\prime}\right)$ that satisfies all degree constraints and that has $w\left(F^{\prime}\right) \leq|C|-2 s$.

First, consider a subset $F^{\prime} \subseteq E^{\prime}$ of the edges in some graph $G_{s}^{\prime}$ such that in $\left(V^{\prime}, F^{\prime}\right)$ every vertex $v$ has degree $d(v)$, and such that $w\left(F^{\prime}\right) \leq|C|-2$ s. If an edge $v^{\prime} v^{\prime \prime}$ is in $F^{\prime}$, then the corresponding vertex $v \in C$ must have one or two $I$-neighbors in $F^{\prime}$. If the edge $v^{\prime} v^{\prime \prime}$ is not in $F^{\prime}$, then the edges $v v^{\prime}$ and $v v^{\prime \prime}$ are both in $F^{\prime}$ and vertex $v \in C$ has no I-neighbors. Denote the sets of vertices $v \in C$ that have zero, one, two $I$-neighbors, respectively by $C_{0}, C_{1}, C_{2}$. Note that $|C|=\left|C_{0}\right|+\left|C_{1}\right|+\left|C_{2}\right|$, that $\left|C_{1}\right|=s$, and that the total weight $w\left(F^{\prime}\right)$ of the edge set $F^{\prime}$ equals $\left(\left|C_{1}\right|+\left|C_{2}\right|\right)-\left|C_{1}\right|=\left|C_{2}\right|$. The condition 


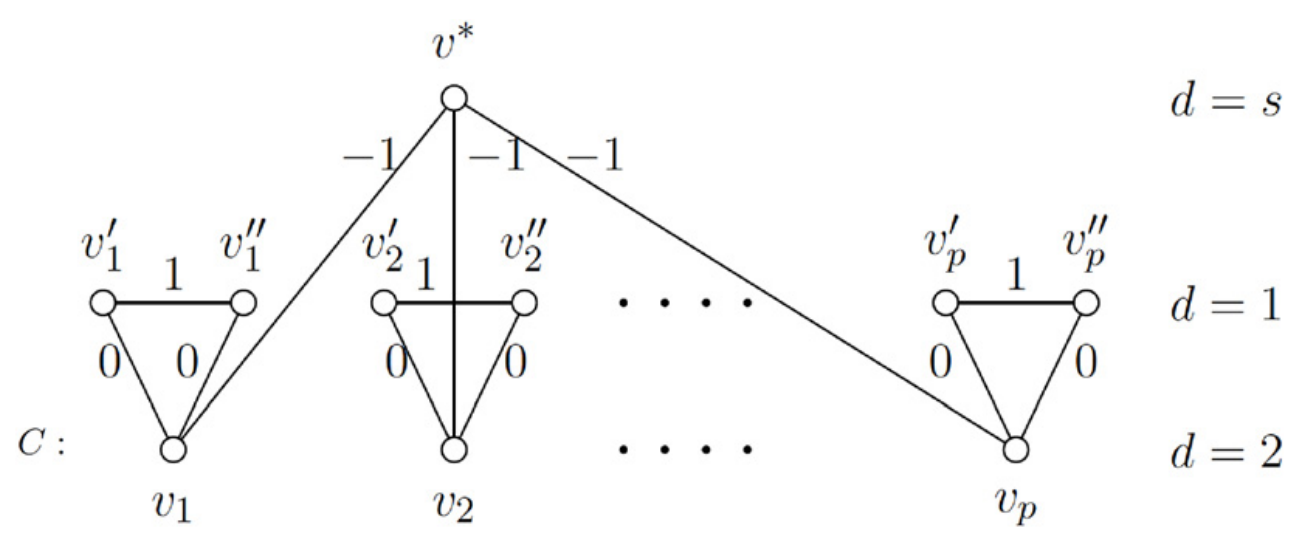

Here are all the $C-I$-edges with weight 0

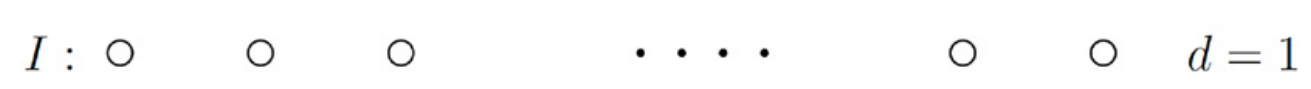

Fig. 5. An instance graph $G_{s}^{\prime}$ in the proof of Lemma 12 .

$w\left(F^{\prime}\right) \leq|C|-2 s$ can be equivalently written as $\left|C_{0}\right| \geq\left|C_{1}\right|$. We group every vertex in $C_{1}$ together with its $I$-neighbor in $F^{\prime}$ and together with an arbitrary vertex from $C_{0}$ into a connected triple. Furthermore, we group every vertex in $C_{2}$ with its two I-neighbors into a triple, and finally we group the remaining unused vertices in $C_{0}$ into triples. The resulting triples form an $\alpha$-decomposition of the split graph $G$ for $\alpha=(3,3, \ldots, 3)$.

Next, assume that $G$ is $\alpha$-decomposable where $\alpha=(3,3, \ldots, 3)$ is the canonical 3-primitive partition of $n$. The triples in this decomposition can be classified into three types: $T_{2}^{1}$-triples have one $C$-vertex and two $I$-vertices; we mark the corresponding two edges between $C$ and $I$. $T_{1}^{2}$ or $\bar{T}_{1}^{2}$-triples have two $C$-vertices and one $I$-vertex; we mark one corresponding edge between $C$ and $I$ (if the $I$-vertex is adjacent to both $C$-vertices, then choose the marked edge arbitrarily). $T_{0}^{3}$-triples have three $C$-vertices; we mark no edges for them. Let $x, y, z$, respectively denote the number of triples of these three types. Note that $x+2 y+3 z=|C|$. If a vertex $v \in C$ is incident to one or two marked edges, then we also mark the edge $v^{\prime} v^{\prime \prime}$. If a vertex $v \in C$ is not incident to any marked edges, then we also mark the two edges $v v^{\prime}$ and $v v^{\prime \prime}$. Finally, if a vertex $v \in C$ is incident to exactly one marked edge, then we also mark the edge $v v^{*}$. It can be verified that for the set $F^{\prime}$ of marked edges, the subgraph $\left(V^{\prime}, F^{\prime}\right)$ satisfies all degree constraints in the graph $G_{y}$. The total weight of $F^{\prime}$ equals $w\left(F^{\prime}\right)=x+y-y=x \leq|C|-2 y$, as desired.

\section{Conclusions}

We have settled the complexity of recognizing fully decomposable split graphs. We feel that it might be very difficult to come up with other graph classes for which this problem is tractable. The algorithm of Barth et al. [1] for recognizing fully decomposable tripodes (trees with a single vertex of degree three, and all other vertices of degree one or two) is highly non-trivial. Unfortunately, many other graph classes contain graphs with a similar connectivity structure as tripodes (with respect to full decomposability); hence settling the problem for these classes would amount to generalizing the proof of [1].

Let us illustrate this claim for the class of cographs. Consider a tripode $T$ that consists of a root and three paths with $\ell_{1}, \ell_{2}$, and $\ell_{3}$ vertices, respectively. We define a corresponding cograph $G(T)$ that consists of three vertex-disjoint cliques with $\ell_{1}, \ell_{2}$, and $\ell_{3}$ vertices, and a single vertex that is joined to all vertices in the cliques. It can be seen that the tripode $T$ is fully decomposable if and only if the cograph $G(T)$ is fully decomposable. We pose the computational complexity of recognizing fully decomposable cographs as an open problem. 
Furthermore, we are not aware of any natural NP-certificates or coNP-certificates for deciding full decomposability of general graphs. In fact, this problem might be located in one of the complexity classes above NP (see for instance Chapter 17 in Papadimitriou's book [9]). If the problem is hard, then the complexity class DP $=\mathrm{BH}_{2}$, the second level of the Boolean Hierarchy, might perhaps be a reasonable guess.

Finally, we will formulate a conjecture that would imply that the problem is easy. Let us call a vector $\alpha$ with positive integer components balanced, if $k-1$ of these components are equal to each other, and the last component does not exceed the other components. We did not manage to construct a counter-example to the following bold conjecture.

Conjecture 13. An n-vertex graph $G$ is fully decomposable if and only if $G$ is $\alpha$-decomposable for every balanced vector $\alpha$ whose components add up to $n$.

If this conjecture turns out to be true (for which admittedly we do not have the slightest evidence), then this would yield an NP-certificate for fully decomposable graphs: there are only $O(n)$ many balanced vectors $\alpha$ whose components add up to the number $n$ of vertices in a graph. The $\alpha$-decompositions for these $O(n)$ vectors form a certificate of polynomial length that can easily be verified in polynomial time.

\section{Acknowledgments}

The first author's work has been supported by EPSRC Grant EP/F064551/1. The third author's research has been supported by the Netherlands Organisation for Scientific Research (NWO), grant 639.033.403, and by BSIK grant 03018 (BRICKS: Basic Research in Informatics for Creating the Knowledge Society).

\section{References}

[1] D. Barth, O. Baudon, J. Puech, Decomposable trees: a polynomial algorithm for tripodes, Discrete Applied Mathematics 119 (2002) 205-216.

[2] D. Barth, H. Fournier, A degree bound on decomposable trees, Discrete Mathematics 306 (2006) 469-477.

[3] H.J. Broersma, D. Kratsch, G.J. Woeginger, Fully decomposable split graphs, in: J. Fiala, J. Kratochvíl, M. Miller (Eds.), IWOCA 2009, in: Lecture Notes in Computer Science, vol. 5874, 2009, pp. 105-112.

[4] M.E. Dyer, A.M. Frieze, On the complexity of partitioning graphs into connected subgraphs, Discrete Applied Mathematics 10 (1985) 139-153.

[5] M.R. Garey, D.S. Johnson, Computers and Intractability: A Guide to the Theory of NP-Completeness, Freeman, San Francisco, 1979.

[6] M.C. Golumbic, Algorithmic Graph Theory and Perfect Graphs, Academic Press, New York, 1980.

[7] M. Horňák, M. Woźniak, Arbitrarily vertex decomposable trees are of maximum degree at most six, Opuscula Mathematica 23 (2003) 49-62.

[8] L. Lovász, M.D. Plummer, Matching Theory, in: Annals of Discrete Mathematics, vol. 29, North-Holland, 1986.

[9] C.H. Papadimitriou, Computational Complexity, Addison-Wesley, 1994. 